\title{
Automatic Detection of ECG Ventricular Activity Waves using Continuous Spline Wavelet Transform
}

\author{
Carlos Alvarado ${ }^{1,2}$, Jesús Arregui ${ }^{2}$, Juan Ramos ${ }^{2}$, Ramon Pallàs-Areny ${ }^{2}$ \\ ${ }^{1}$ Bioelectronics Section, Department of Electrical Engineering, CINVESTAV-IPN, México D.F., México \\ ${ }^{2}$ Department of Electronic Engineering, Technical University of Catalonia (UPC), Barcelona, Spain \\ Tel: (55) 5061-3800 Ext. 6216 Fax: (55) 5061-3981 Email: calvarad@cinvestav.mx
}

\begin{abstract}
In this study we present detection algorithms of characteristic points of the QRS and $T$ waves based on the continuous wavelet transform (CWT) with splines. This technique can handle any integer scale and the analysis is not restricted to scales that are powers of two, which allows to use a wide range of scales and to more efficiently reduce noise and artifacts. Evaluation of the QRS detection algorithm performance has been done in eight ECG data files of the MITBIH database, and the accuracy has been of $99.5 \%$. Evaluation of the detection algorithms of the QRS wave onset and offsets of QRS and T waves has been done in the CSE multi-lead measurement database, and the measurements were within the tolerance limits for deviations with respect of the manual measurements determined by the CSE experts. Therefore, the proposed algorithms to detect characteristic points of the QRS and T waves based on this technique allow the evaluation of the CWT in more scales, are robust to noise and artifacts and have the accuracy of a human expert.
\end{abstract}

Keywords - Detection algorithms, ECG, wavelet transform, splines

\section{INTRODUCTION}

Measurements of ECG time intervals like QT interval are important in electrocardiographic diagnosis because reflect electrophysiological processes and are good indicators of cardiovascular diseases when they lie outside the range of the normal variation. The accurate detection of the QRS complex in the presence of noise is the most important task in the ECG automatic analysis, because when it has been detected a more detailed examination of other ECG waves and segments can be performed.

Many techniques mostly nonsyntactic have been used for QRS detection [1-4]. These techniques mainly suffer from two problems: 1) the bandwith of the QRS complex is different for different subjects and even for different beats of the same subject; and 2). The bandwith of the noise and of the QRS complex overlap. For QT interval measurement, several techniques have been reported, and the most difficult problem is to detect the T-wave end because of its low frequency components [5-7]. Wavelet transforms (WT) are commonly used to identify ECG waves because of their ability to detect transients and also because of their robustness in front of noise and artifacts [8,9].
Wavelet transforms at different scales describe the time characteristics of a signal in different frequency bands, but analysis is restricted to scales that are powers of two [10]. Using B-splines as base functions allow the evaluation of the continuous wavelet transform (CWT) in any integer scale [11], which allows to use a wide range of scales and to more efficiently reduce noise and artifacts. In this study, we present detection algorithms of characteristic points of the QRS and T waves based on the CWT with splines, that have been evaluated with ECG records of the MIT-BIH and the CSE multi-lead measurement databases.

\section{WAVELET TRANSFORM}

Wavelet transforms yield the components of a signal in different scales [10]. The CWT of a time-continuous signal $x(t)$ is defined by the convolution integral

$$
\operatorname{CWTx}(b, a)=\frac{1}{\sqrt{a}} \int_{\alpha}^{\infty} X(t) \psi^{*}\left(\frac{t-b}{a}\right) d t
$$

where $\psi(t)$ is the wavelet function and $a$ and $b$ are, respectively, the scale and translation parameters. $\psi(t)$ compresses or dilates depending on $a$, which allows the CWT to extract the low- and high-frequency components of $x(t)$. To implement the CWT, $a$ and $b$ are usually discretized. If $a$ is discretized over a sequence $2^{j}(j=1,2, .$.$) , the analysis$ is restricted to scales that are powers of two, and we obtain the dyadic wavelet transform that can be computed with Mallat's algorithm [10].

In this study, we use B-splines instead that allow the evaluation of the CWT in any integer scale $[11,12]$. In this approach, the input signal and the analyzing wavelet are both represented by polinomial splines of degree $n_{1}$ and $n_{2}$ respectively, and the resulting CWT at scale $\mathrm{m}$ is a polynomial spline function given by

$$
\operatorname{CWT}(x(t), m, k)=\sum_{k \in Z}\left([p] \uparrow_{m} * u_{m}^{n_{2}} * b^{n_{1}+n_{2}+1} * c\right)(k)
$$


where the notation $[p] \uparrow_{m}(k)$ represents the upsampling of the sequence $p$ by a factor of $m, b^{n 1+n 2+1}$ is the $B$-spline representation of a spline of order $\left(n_{1}+n_{2}+1\right)$ and the filter $\mathrm{u}_{\mathrm{n} 2, \mathrm{~m}}$ is equivalent to a cascade of $\left(n_{2}+1\right)$ filters of moving average of order $(m-1)$ with a offset $k_{0}$ that ensures that $\mathrm{u}_{\mathrm{n} 2, \mathrm{~m}}$ is symmetrical. The SPWAV program developed is based in the fast algorithm proposed by Unser et al. [11], and calculates the CWT of the discrete signal $x(t)$ at the integers scales $m$ with expansion coefficients spline $p$ [12].

The selected wavelet function $\psi(t)$ is the first derivative of a cubic B-spline of $4^{\text {th }}$ order expanded by two (Fig. 1). This wavelet function is similar to the first derivative of a Gaussian function, which yields good time and frequency resolution.

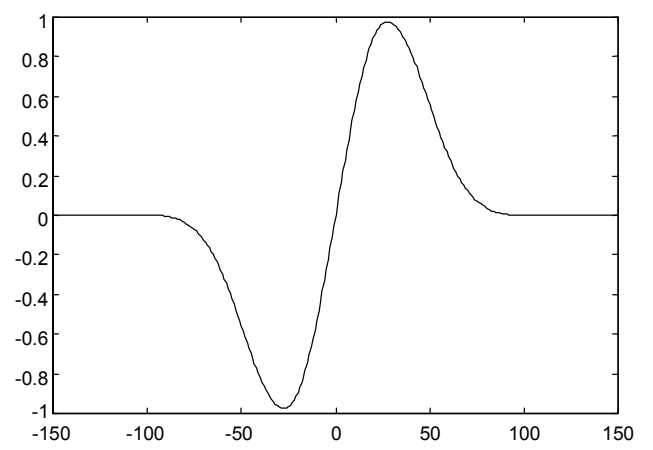

Fig. 1. First derivate of a fourth-order B-spline expanded by a factor of two.

The Fourier transform of the wavelet at five scales are shown in Fig. 2, and their $-3 \mathrm{~dB}$ bandwiths at a sampling frequency of 500 samples per second are listed in table 1.

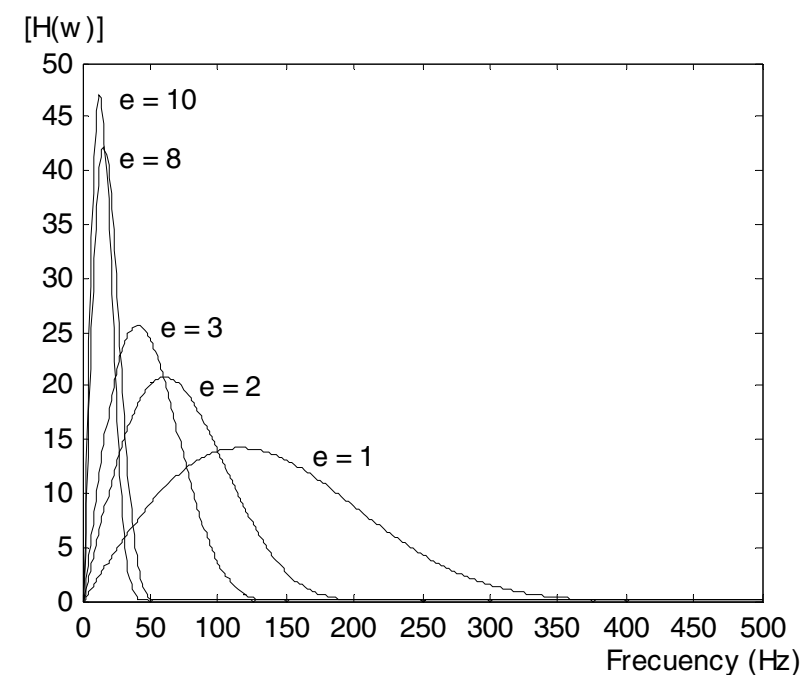

Fig. 2. Amplitude-frequency responses of equivalent filters at five scales corresponding to $500 / \mathrm{s}$ sampling rate.
Table 1. Frecuency response of equivalent filters at five scales.

\begin{tabular}{|c|c|}
\hline Scale (e) & $\mathbf{- 3}$ dB bandwidth $\mathbf{~ H z )}$ \\
\hline 1 & $56-186$ \\
\hline 2 & $30-97$ \\
\hline 3 & $19-64$ \\
\hline 8 & $7-24$ \\
\hline 10 & $6-19$ \\
\hline
\end{tabular}

\section{DETECTION METHODS}

\section{1) $Q R S$ detection}

Because of the wavelet function selected, each distinct wave of the ECG corresponds to a pair of local maxima of the modulus (Pmm) of the CTW at each different scale; the rising slope of each wave yields a minimum and the falling slope yields a maximum [8]. The R-wave peak, (Rp), corresponds to the zero crossing observed between the Pmm (Fig. 3).
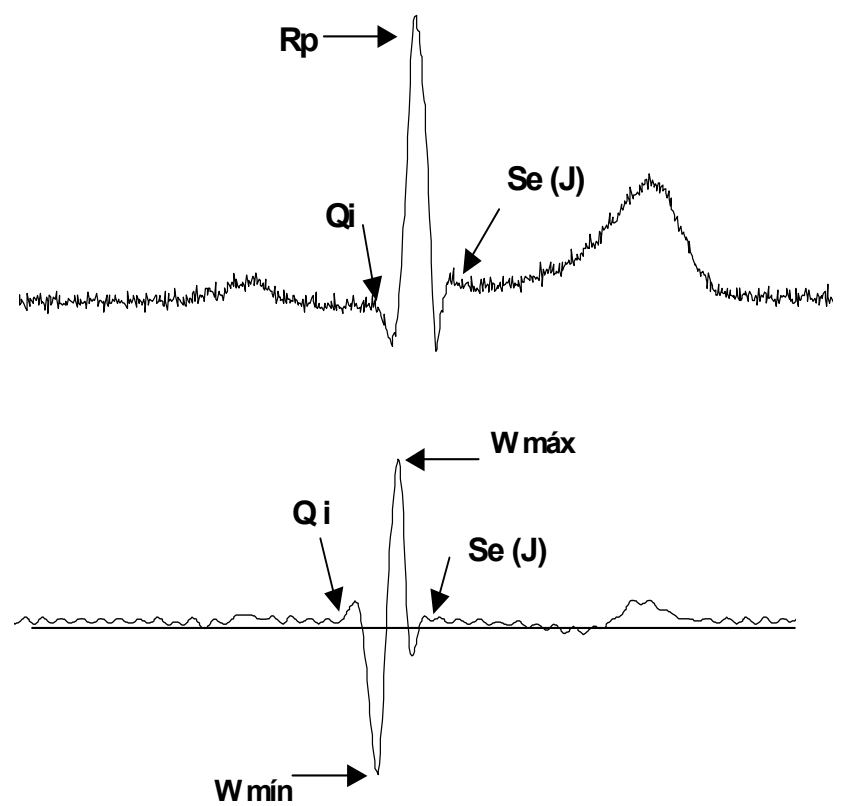

Fig. 3. ECG (top) and characteristic points of the wavelet transform of the QRS complex at two scale (bottom).

The developed algorithm detects the QRS by using the scale 2 and the Pmm corresponding to the R wave by threshold comparing inside a search window defined by the average $\mathrm{RR}$ interval and the last RR interval calculated [5]. From that Pmm, the start of the Q wave Qi (or the R wave in the absence of $\mathrm{Q}$ wave) corresponds to the zero crossing preceding the Pmm; the end of the S wave Se (or the end of the $\mathrm{R}$ wave in the absence of the $\mathrm{S}$ wave) corresponds to the zero crossing after the Pmm. 
Those zero crossings are detected by looking inside a search window defined by the maximal duration of both waves.

\section{2) T-wave detection}

The developed algorithm applies only to normal and inverted $\mathrm{T}$ waves. Baseline drifts and artifacts are removed by using the scale 10 of the WT. The T wave is detected as follows: from $\mathrm{Rp}$ we define a search window whose length decreases when RR diminishes [5]; inside that window we look for the Pmm corresponding to the $T$ wave. The location of the Pmm correspond to, respectively, the beginning (Ti) and the end (Te) of the $\mathrm{T}$ wave. Because of the symmetry of the normal $T$ wave, its peak (Tp) corresponds to the zero crossing placed between the Pmm (Fig.4).
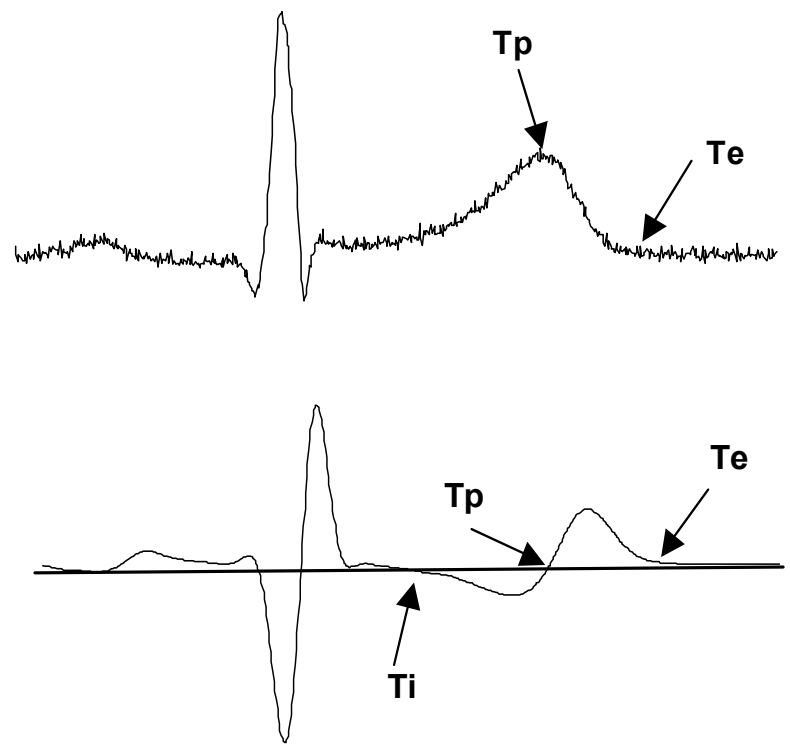

Fig. 4. ECG (top) and characteristic points of the wavelet transform of the T wave at ten scale (bottom).

\section{RESULTS}

\section{1) $Q R S$ detection}

The algorithm for QRS detection has been first tested on eight $30 \mathrm{~min}$ recordings from the MIT-BIH arrhythmia database [13], in where only channel 1 of the two-channel ECG recordings was used. The selected recordings included serious noise bursts, baseline drifts and movement artifacts.

Table 2 shows that we had 81 false QRS detections $(0.47$ $\%)$; 51 of them were false positives and 30 were false negatives.

\section{2) Detection of characteristic points of the QRS and T waves}

The proposed algorithms to detect the beginning (Qi) and the end (Se) of the QRS, and the end of the T wave (Te) have been tested on 25 recordings from the CSE database [14], that includes 15 ECG leads and manual annotations on them [15].

Table 2. Validation results for the proposed QRS detection algorithm applied to eight records from the MIT-BIH.

\begin{tabular}{|c|c|c|c|c|c|}
\hline \multirow{2}{*}{$\begin{array}{c}\text { ECG } \\
\text { record } \\
\text { number }\end{array}$} & Beats & F.P. & F.N. & \multicolumn{2}{|c|}{} \\
\cline { 5 - 6 } & & & & Beats & \% \\
\hline \hline 100 & 2272 & 0 & 1 & 1 & 0.04 \\
\hline 101 & 1864 & 0 & 1 & 1 & 0.05 \\
\hline 102 & 2187 & 0 & 0 & 0 & 0 \\
\hline 103 & 2084 & 0 & 0 & 0 & 0 \\
\hline 104 & 2229 & 17 & 4 & 21 & 0.9 \\
\hline 105 & 2571 & 31 & 13 & 44 & 1.71 \\
\hline 107 & 2135 & 0 & 1 & 1 & 0.04 \\
\hline 108 & 1753 & 3 & 10 & 13 & 0.7 \\
\hline Total & $\mathbf{1 7 0 9 5}$ & $\mathbf{5 1}$ & $\mathbf{3 0}$ & $\mathbf{8 1}$ & $\mathbf{0 . 4 7}$ \\
\hline
\end{tabular}

F.P. false positives, F.N. false negatives

Table 3 shows the average $(m)$ and standard deviation (sd) of the difference between the (WT-based) automatic and the (CSE) manual (annotated) location of those characteristic points. The results for that difference are within the tolerance limits accepted in the CSE database [16].

Table 3. Validation results for automatic detection of characteristic points Qi, Se and Te on annotated recordings of the CSE database.

\begin{tabular}{|c|c|c|c|}
\hline \multirow{2}{*}{$\begin{array}{c}\text { Recordings CSE } \\
\text { Mo1_001:121 (5:5) }\end{array}$} & Qi & Se & Te \\
\cline { 2 - 4 } & WT - CSE & WT - CSE & WT - CSE \\
\hline \hline m \pm sd & $-4.5 \pm 1.5$ & $7.6 \pm 1.8$ & $8.2 \pm 3.6$ \\
\hline \multicolumn{4}{|c|}{ Tolerance limits for deviations with respect to experts } \\
[CSE 1985]
\end{tabular}

Values are in milliseconds; m, mean; sd, standard deviation. 


\section{CONCLUSIONS}

The proposed algorithms to detect characteristic points of the QRS and T waves based on the continuous wavelet transform with splines can handle more scales that the dyadic wavelet transform because they are not restricted to scales that are powers of two. This characteristic allows reduce effects of baseline drifts and artifacts mainly in the detection of peak and end of $\mathrm{T}$ wave using the scale 10 of the WT. Therefore, the algorithms are robust to noise and artifacts, have the accuracy of a human expert and allow the determination of ventricular activity intervals clinically useful for cardiac diagnosis.

\section{ACKNOWLEDGEMENTS}

This work has been funded by a scholarship from the CONACYT (México) to Carlos Alvarado and by project SAF98-0120 from the Spanish CYCIT.

\section{REFERENCES}

[1] Q.Z. Xie, Y.H. Hu, W.J. Tompkins, "Neural-network based adaptive matched filtering of QRS detection", IEEE Trans. Biomed. Eng., vol. 39, 317-329, 1992.

[2] F. Gritzali, G. Frangakis, G. Papakonstantinou, "Detection of the $\mathrm{P}$ and T waves in an ECG", Comput. Biomed. Res., vol. 22, pp. $83-91,1989$.

[3] J. Pan. and W.J. Tompkins, "A real-time QRS detection algorithm", IEEE Trans. Biomed. Eng., vol. 32, 230-236, 1985.

[4] P.S. Hamilton and W.J. Tompkins, "Quantitative investigation of QRS detection rules using the MIT/BIH arrhythmia database", IEEE Trans. Biomed. Eng., vol. 33, pp. 1157-1165, 1986.
[5] P. Laguna, N.V. Thakor, P. Caminal, R. Jané, H.R. Yoon, A Bayés de Luna, V. Marti, J. Guindo "New algorithm for QT interval analysis in 24-hour Holter ECG: performance and applications", Med. \& Biol. Eng. \& Comput., vol. 28, pp. 67-73, 1990.

[6] A. Algra, H. Le Brun, C. Zeelenberg, "An algorithm for computer measurement of QT intervals in the 24 hour ECG", in Computers in Cardiology, IEEE Computer Society Press, pp. 117-119, 1987.

[7] G. Critelli, F. Marciano, M. Mazzarella, M.L. Migaux, "QT interval measurement of long term ECG recordings. Application to an automatic Holter analysis system", in Computers in Cardiology, IEEE Computer Society Press, pp. 480-481, 1982.

[8] C. Li, C. Zheng, C. Tai, "Detection of ECG characteristic points using wavelet transforms", IEEE Trans. Biomed. Eng.., vol. 42, no. 1 , pp. 21-28, 1995.

[9] J.S. Sahambi, S.N. Tandon, R.K.P. Bhatt, "Using wavelet transforms for ECG characterization", IEEE Eng. Med. Biol. vol. 16 , no. 1 , pp. 77-83, 1997.

[10] S. Mallat, "A theory for multiresolution signal decomposition: the wavelet representation", IEEE Trans. Patt. Anal. Mach. Intell., vol. 11, no. 7, pp. 674-693, 1989.

[11] M. Unser, A. Aldroubi, S.J. Schiff., "Fast implementation of the continuos wavelet transform with integer scales", IEEE Trans. on Signal Processing, vol. 42, no. 12, pp. 3519-3523, 1994.

[12] J. Arregui, "Study of spectrotemporal methods and their application to the ECG", Final Project, ETSETB, Universitat Politècnica de Catalunya, Barcelona, Spain, 1996. (In Catalan)

[13] G.B. Moody and R.G. Mark, "The MIT-BIH arrhythmia database on CD-ROM and software for use with it", in Computers in Cardiology, IEEE Computer Society Press, pp. $185-188,1990$.

[14] J.L. Willems, et al. for the CSE Working Party, "Common standards for quantitative electrocardiography: goals and main results", Meth. Inform. Med., vol. 29, pp. 263-271, 1990.

[15] J.L. Willems, Common standards for quantitative electrocardiography. CSE multilead atlas, Measurement resultsdata set 3. CSE Project, Leuven, Belgium, ACCO Publ.; 1988.

[16] The CSE working party, "Recommendations for measurement standards in quantitative electrocardiography", Eur. Heart J., vol. 6 , pp. 815-825, 1985 . 